\title{
Dipole-Switch Induced Modification of the Emissive Response of Carbon Nanotubes
}

\author{
M Glaeske $^{1 *}$, P Bluemmel ${ }^{1}$, S Juergensen ${ }^{1}$, A Setaro ${ }^{1 *}$, and S Reich ${ }^{1}$ \\ ${ }^{1}$ DePartment of Physics, Freie UniversitÄt Berlin, ARnimalleE 14, 14195 Berlin
}

\begin{abstract}
The interaction of carbon nanotubes with the molecular dipole switch spiropyran is expected to affect the optical response of the tubes. Until now, the need of anchor groups to immobilize the switches on the tubes has hindered experimental observation of effects of switching on the emission behavior of the tubes. Here we present spiropyran-carbon nanotubes complexes obtained by micelle swelling. This method does not require any anchor nor sophisticated chemistry to warrant close tubeswitch proximity. For the first time we observe the shifts predicted theoretically and their effect on the tubes' excitation and emission energies.
\end{abstract}

*corresponding authors: mareen.glaeske@fu-berlin.de, setaro@physik.fu-berlin.de 


\section{Introduction}

The functionalization of carbon nanotubes (CNTs) with molecular moieties creates nanohybrids with new outstanding properties. By choosing the proper functionality, the characteristic features of CNTs can be altered in a controlled way, enabling new, sophisticated technological applications, e.g. switching of the conductance [1], emission enhancement [2] and energy transfer [3],[4]. In particular, the functionalization of carbon nanotubes with photochromic moieties, such as spiropyran, has been of great interest. They can be exploited for engineering sensors [5] and memory devices [5]-[7]. Spiropyran is a photochromic molecule consisting of a benzopyran and an indole moiety connected via a $\mathrm{sp}^{3}$ hybridized carbon atom. For an overview of the properties of spiropyran, please refer to Ref. [8]. Spiropyran can be reversibly switched between the open merocyanine (MC) and the closed spiropyran (SP) conformation. During the isomerization process, driven by ultraviolet light, the carbon-oxygen bond breaks, converting the spiro center to an $\mathrm{sp}^{2}$ hybridized carbon atom, leading to a co-planar arrangement of the benzopyran and indole moieties in the merocyanine form with the $\pi$-electron delocalized along the entire molecule, generating an absorption band in the visible around 590nm. The newly formed merocyanine is an extended $\pi$-conjugated system with a zwitterionic character. It is much more polar then the SP form, giving rise to a large dipole moment.

The immobilization of the photochromic switches on the CNTs surface can be realized through different approaches, such as non-covalent functionalization, polymer wrapping and covalent functionalization [7],[9]-[12]. Here we focus on a new and simple functionalization approach which does not require sophisticated chemistry - the micelle swelling technique [13]. It allows investigating the interaction of molecular moieties when they lie in the close proximity of CNTs without the need of specialized surfactants comprising both CNT debundling and switchability [14]-[16].

The coupling of the molecular dipole moment with the excitons of the CNTs is expected to strongly affect the optical properties of the CNTs. Malic et al. predicted a shift of several meV in the exciton transition energies [17],[18]. Furthermore they investigated different tube-switch configurations and showed that the largest shifts are observed for a dipole orientation of $90^{\circ}$ perpendicular to the tubes' axis and optimal switch coverage of $0.25 \mathrm{~nm}^{-1}$. The Coulombic dipole-dipole interaction decreases with an increase in relative switch-tube distance; hence a close proximity is aimed to observe the strongest shift. The dipole distribution, on the other hand, has no measurable effect on the transition energies. At the same concentration, randomly and homogeneously distributed dipoles provide the same shifts of the excitonic energies.

In our previous studies, we verified the theoretical predictions by Malic et al. and experimentally observed reversible shifts of the absorption bands of specially engineered SP-Pyrene molecules which 
were non-covalently anchored onto the tubes via $\pi-\pi$ stacking of the pyrene part [7],[19]. In these studies, the limiting factor to fully exploit the dipole-dipole interaction was the CNT-switch separation, which could not be made small at will, as in this approach it is limited by the necessity of an anchor group to be conjugated to the switch part [20], [21]. The studies showed that the interaction between molecular switches and CNTs increased with decreasing their distance. The most significant effects, however, were observed on the merocyanine (with strong shifts of its absorption bands and an increase of the merocyanine lifetime with respect to the spiropyran form) and not on the tubes.

Here we consider micelle swelling as a technique to minimize the tube-switch distance. It does not require any anchoring group to ensure the proximity of the switch to the nanotubes sidewall. Micelle swelling was introduced by Wang et al. [13] and established a new route to investigate the interaction of molecular moieties and CNTs. Roquelet et al. exploited it to transport the organic molecule porphyrin within the micelles solubilizing tubes, studying the energy transfer between porphyrins and CNTs [22]. Kreft et al. demonstrated that micelle swelling could be used to carry the orthogonal switchable molecules (DHA/VHF) into the micelles of the CNTs while retaining the molecule switching ability [23]. Micelle swelling can be adapted to create plasmonic hybrids comprising metallic nanoparticles and CNTs [2],[24] and to create nano-reactor environments to trigger polymerization around the CNTs to establish a bridge between covalent and non-covalent functionalization of the tubes [25].

Micelle swelling is beneficial in several ways; the micellar forces ensure the tubes and the switches to be confined within the hydrophobic core, providing direct proximity between nanotube and switches. It moreover provides excellent solubilization of the tubes and allows to control the switch-to-tube ratio within the micelles and thus the nanotube coverage by the switches. Figure 1 schematically depicts the micelle swelling process. Molecular agents that are insoluble in water are solubilized in organic solvents and added to a solution with CNTs suspended in surfactant micelles, Fig. 1a. While stirring, the molecular agents are transported by the organic solvent into the hydrophobic environment of the

a)

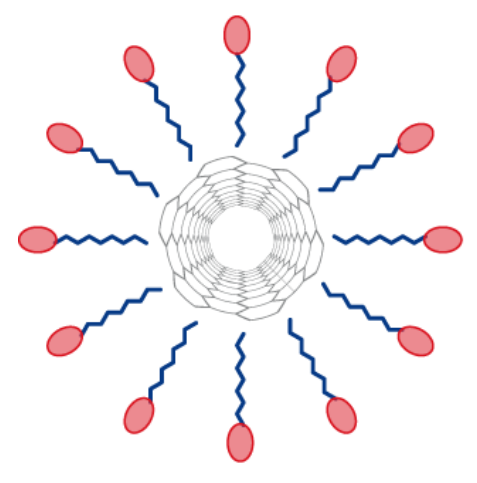

b)

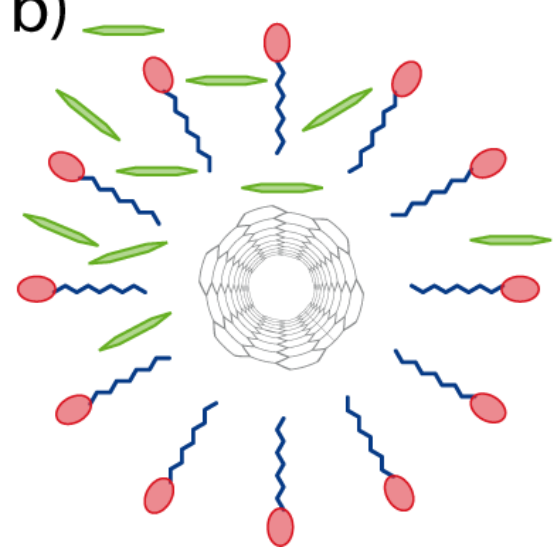

c)

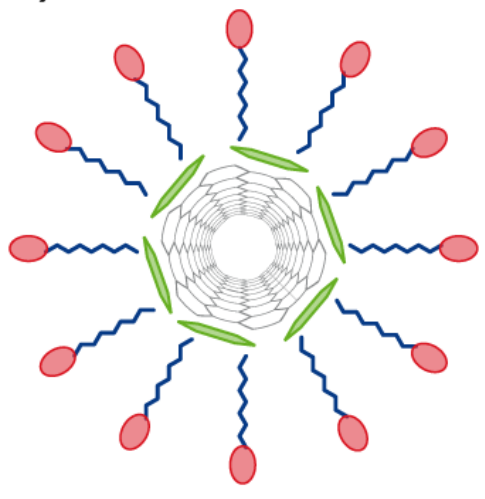

Figure 1 Scheme of the micelle swelling approach: a) micelle suspended CNTs in water. b) After the addition of the organic solvent containing the molecule, both start to penetrate the micelle. c) The solvent will evaporate and the molecules will be encapsulated within the micelle core and arrange themselves around the CNTs. 
micelles' inner-core, Fig 1b. After a while, the organic solvents evaporate and the molecules are enclosed within the micelles already containing the CNTs, Fig. 1c. The micelle swelling thus ensures a strong interaction between CNTs and molecular switch, which increases the dipole-tube interaction compared to our previous studies, ensuring a contiguous position of the switch on the nanotubes surface

\section{Experimental}

CNTs were dispersed in SDS as described in Refs. [26] and [27]. A solution of CoMoCAT tubes $(0.1 \mathrm{~g} / \mathrm{L})$ were dispersed in water with $1 \mathrm{wt} \%$ SDS and sonicated for $1 \mathrm{~h}$ at $20 \mathrm{~W}$, followed by centrifugation at $31000 \mathrm{~g}$. The prepared stock solution and was used for preparing all of the samples to ensure identical amount of tubes in all experiments. To transport molecules into the tubes-containing micelles, $150 \mu \mathrm{L}$ of THF yielding, respectively, $0,50,100,150 \mu$ g spiropyran were added to $3 \mathrm{~mL}$ of nanotube stock solution. The mixture was stirred for $48 \mathrm{~h}$ in a water quench at room temperature.

For the optimization study, the temperature was adjusted to $30^{\circ} \mathrm{C}, 45^{\circ} \mathrm{C}$ and $55^{\circ} \mathrm{C}$. The temperatures have been chosen to be lower than the boiling point of the solvent to avoid its evaporation before the switches are inside the micelles. After the swelling procedure, the samples were centrifuged for $10 \mathrm{~min}$ to remove bundles that might have been formed or tubes no longer enclosed by a micelle. The samples were divided into two groups: One set was prepared in complete darkness (we will refer to them as the DARK samples in the rest of this manuscript) while the other set was prepared under UV irradiation with a 366nm lamp (we will refer to this as the UV sample). In the case of the UV samples, the switch solution was irradiated already before adding it to the SWNT suspension. In this way we ensure that the molecules entering the micelles in the DARK samples were mostly in their SP form while in the UV samples were mostly in the MC form. Please note that, while irradiating the samples with UV light, we reach a photostationary state that is given by the balance between the SP-to-MC light induced isomerization and the MC-to-SP thermally induced back isomerization. The value of this ratio is the highest conversion rate we may achieve at the working temperatures and in the given solvent. Thus, half of the final samples (the UV ones) were tubes coated with merocyanine and the other half (the DARK ones) with spiropyran. 

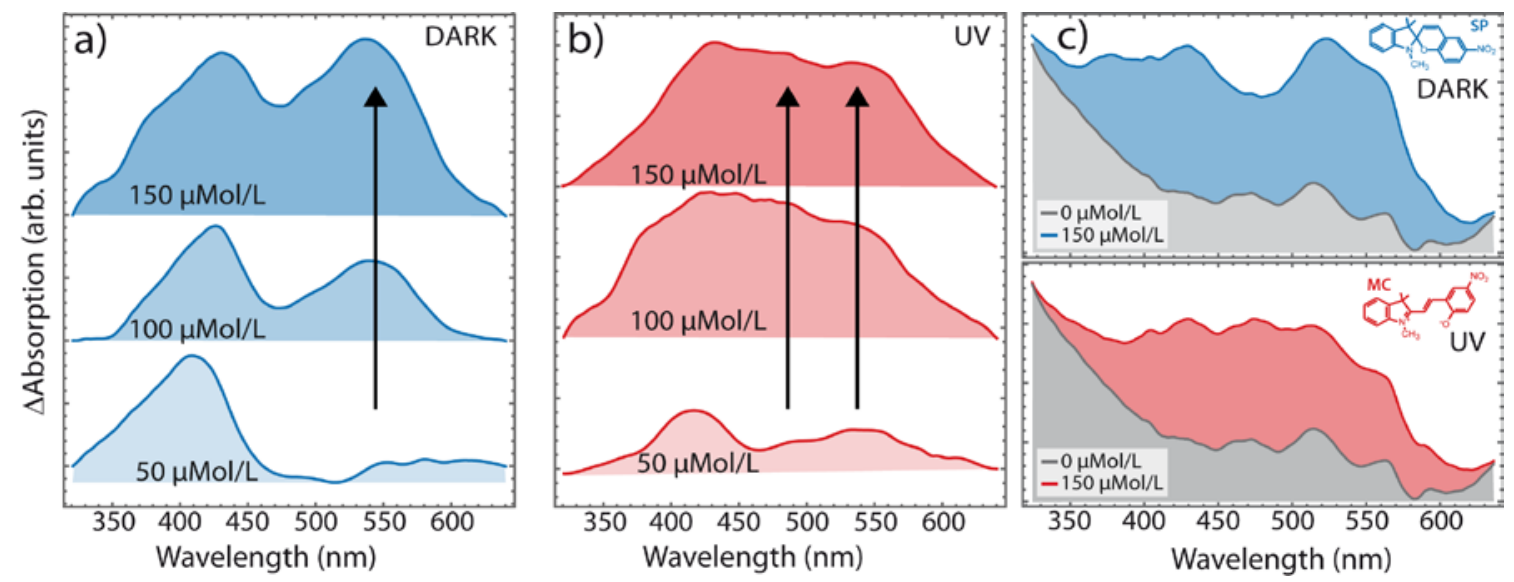

Figure 2 Differential absorption spectra (reference spectrum: Pristine CNTs) of the DARK a) and UV samples b) with different switch concentrations. c) Absorption spectra of the $150 \mu \mathrm{Mol} / \mathrm{L}$ samples in comparison to the pristine tubes.

\section{Results and Discussion}

\section{Effect of the dipole coverage}

As predicted by Malic et al., the dipole-dipole interaction between the MC and the tubes leads to red shifts in the transition energies of the nanotubes. Up to now, the only experimental evidence of such shift were changes of the absorption bands of the carbon nanotubes [17],[20],[21]. It was impossible to conduct photoluminescence excitation (PLE) measurements of the tubes coated with MC, as the excitation radiation (500-800nm) required for performing a PLE map causes the back isomerization of MC into SP [9],[20],[21]. Absorption measurements, on the contrary, require less intensities; they do not trigger back isomerization and let us observe the influence of the dipole moment on the absorption bands of the tubes. The micelle swelling approach, however, offers the advantage to stabilize the state of the switch inside the micelle and to lock its state. The switch remains in its configuration and back isomerization is inhibited, making it possible to study the emission energies of CNTs decorated with spiropyran switches in both the SP and the MC form. Previous studies have shown that the lifetime of the merocyanine form gets longer as it gets closer to the nanotube sidewall, due to the increased $\pi-\pi$ interaction between the merocyanine $\pi$-electron and the tubes[9],[20],[21]. If merocyanine is directly adsorbed onto the nanotube surface, then it is expected to be locked in its state without isomerizing back. Such a behavior was already observed for MC on a gold surface [28].

As the theoretical predictions suggest optimal dipole-dipole interaction for specific dipole coverage, we varied the SP/MC switch concentrations in the micelle swelling to verify the effect of the coverage onto the optical response of the tubes. Figure $2 a$ ) and b) depict the absorption spectra for the different switch concentrations in darkness and under UV radiation, respectively, identifying the state of the switch. The characteristic MC peaks at 390nm and 500nm are observed in both the DARK (Fig.2a) and 
the UV (Fig.2b) sample. The increase in switch concentration shows an increase in the bands intensities. The absorption of the UV sample includes an additional peak around $400 \mathrm{~nm}$, which is associated with stacked MC [29]. It is interesting to note that Fig. 2a) indicates the presence of the MC peak even in the DARK sample, which should only exhibit the ring-closed SP form. The micelle swelling process inserts the molecules into a highly complex environment made of carbon nanotubes, water, THF and SDS. This presumably affects the SP/MC equilibrium point and leads to such behavior. As water is polar, the hydrophobic SP is trapped in the special limited hydrophobic tube-micelle environment. This special environment and the limited space between the micelle and the tube can lead to the presence of MC in the DARK sample.

Albeit the locked MC state and the presence of the MC in the DARK sample indicates tube-switch interaction, the main goal of this study was to observe the influence on the transition energies of the CNTs for both the excitation and the emission of the tubes. The PLE measurements show clear differences between the UV and the DARK samples. A first striking feature is that, with increasing

a)

b)
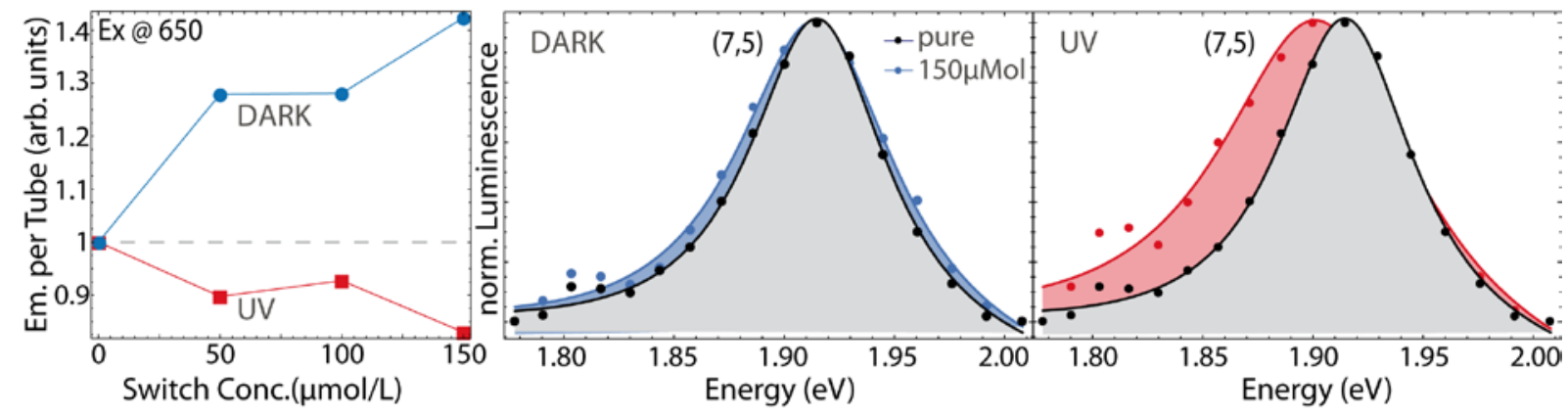

c)

DARK

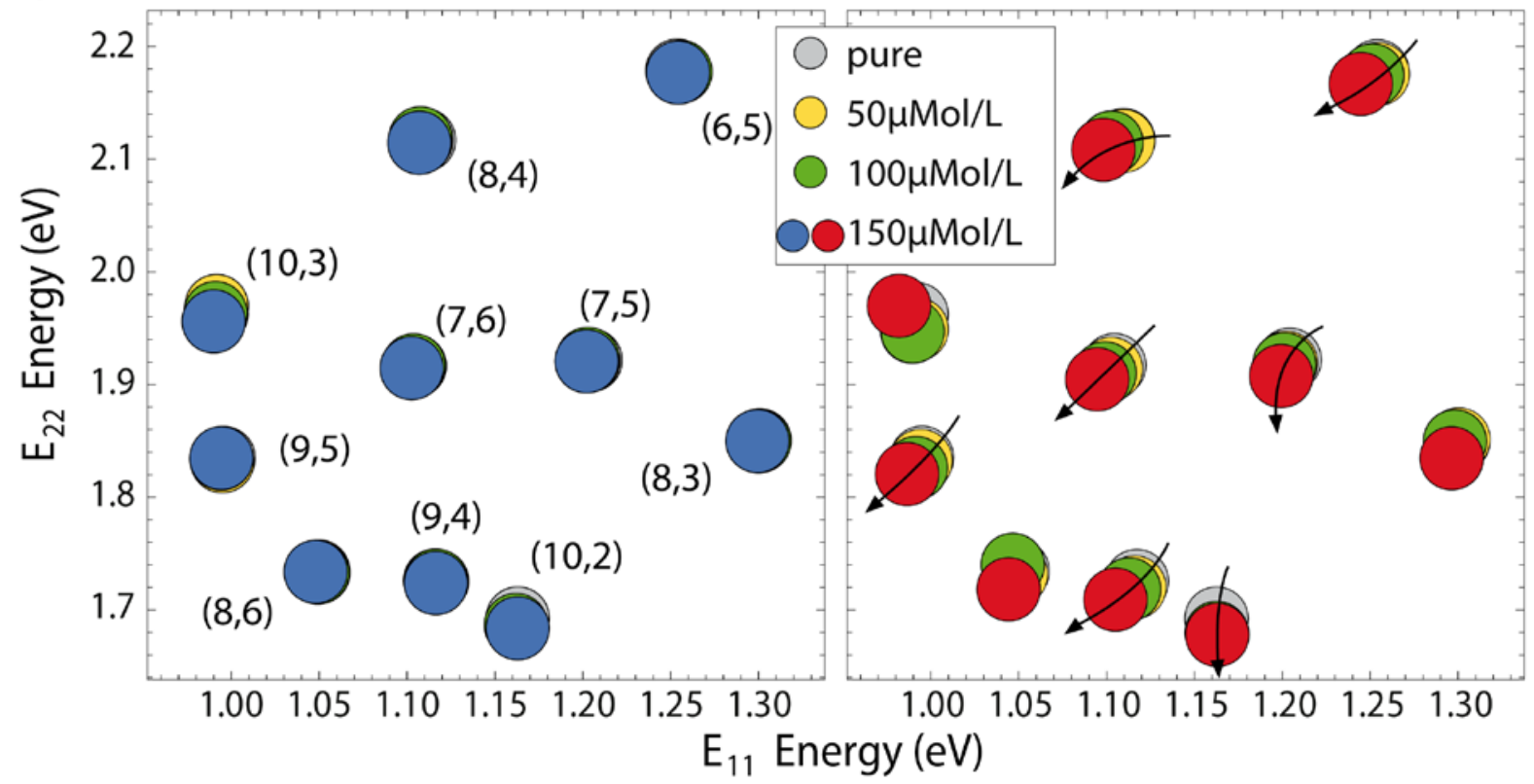

Figure 3 a) Average luminescence of the tubes excited at 650nm, obtained by the emission peak areas divided by the area of the absorption band after background removal. b) Emission of the $(7,5)$ tube in the DARK sample and emission of the $(7,5)$ tube of the UV irradiated sample. c) Pseudo PLE map of all the tubes left of the DARK sample, right of the UV sample (an excerpt of the PLE map is published in [31]). 
switch concentration, the emission yield from the DARK samples rises. The UV samples exhibit the opposite behavior with a decrease in tubes emission with increasing switch concentration. This behavior is depicted for the $(7,5)^{1}$ tube in Figure 3a). We believe that the increase of emission of the DARK sample is due to the fact that the SP, which is a non-planar moiety, promotes exfoliation of small nanotube bundles and yield their further debundling, in a similar fashion as cholate derivatives do exfoliate the tubes. The interaction between strong MC dipoles within the micelle core and the ionic head of the surfactant could lead to destabilization of the micelles, resulting in a loss of tubes.

The presence of the switch, though, does not only affect the overall intensity emitted from our samples. As predicted by the theory, the dipole-dipole interaction of the switch and the CNTs changes the exciton energies of the tubes. The emission by the $(7,5)$ tube species in Figure $3 b)$ demonstrates that the transistion energies in the DARK sample remain constantly. The points scatter by $2 \mathrm{meV}$ around the pristine nanotube position, for both emission and excitation energies. The UV sample, on the other hand, exhibits a shift of $9 \mathrm{meV}$ for the $\mathrm{E}_{11}$ transition (emission energies) and $12 \mathrm{meV}$ for the $\mathrm{E}_{22}$ transition (excitation energies). This trend is also observed for the other tubes. The pseudo PLE map in Fig. 3c) compares the transition energies for the DARK and UV samples. The tubes in the DARK sample hardly show any peak shift with increasing switch concentration. Contrary, the UV samples exhibit diverse shifts for different nanotube chiralities and switch concentrations as highlighted by the bent arrows in the right panel. The fact, that the shift increase with higher switch coverage of the tubes indicates that the given switch concentration is still below the optimal dipole coverage of $0,25 / \mathrm{nm}$. When surpassing this value lateral dipole-dipole interactions start interfering with the dipole-exciton interaction, leading to a decrease of the effective switch influence on the transition energies of the tubes. This phenomenon was experimentally observed by Kreft et al. for the switching molecule DHA/VHF and reported in reference [23]: The shifts increase by increasing the coverage of the tube and, after reaching a critical coverage, the shift will decrease. In our case, we observed a monotonous trend of continuous increase of the shift intensity by increasing the switch concentration and do not observe reduction of the shifts. Providing an experimental demonstration of the changes in excitation and emission energy for carbon nanotubes decorated with SP/MC, as predicted by Malic et al. [17].

\section{Effect of the dipole intensity}

To further confirm the theoretical predictions, we monitored the shifts of the exciton energies for dipole switches with varying dipole moment. We compared the dependence of the energy shifts on the magnitude of the dipole moment. To this purpose, we use two different derivatives of the spiropyran

1 CNTs are cylinders made of rolled graphene sheets. The different species are each described by its characteristic chiral vector $\left(n_{1}, n_{2}\right)$. 
a) 7

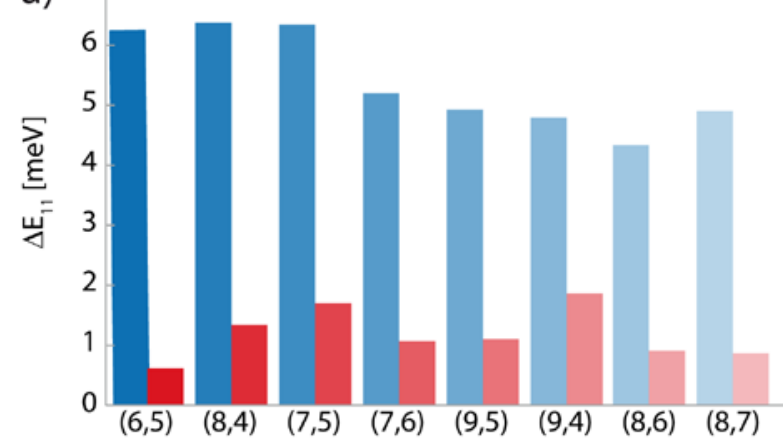

c)

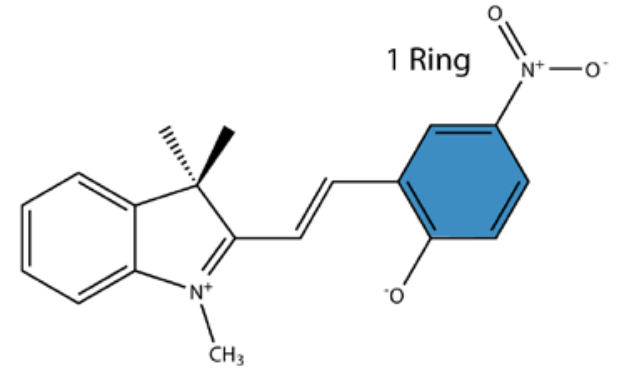

b) 14

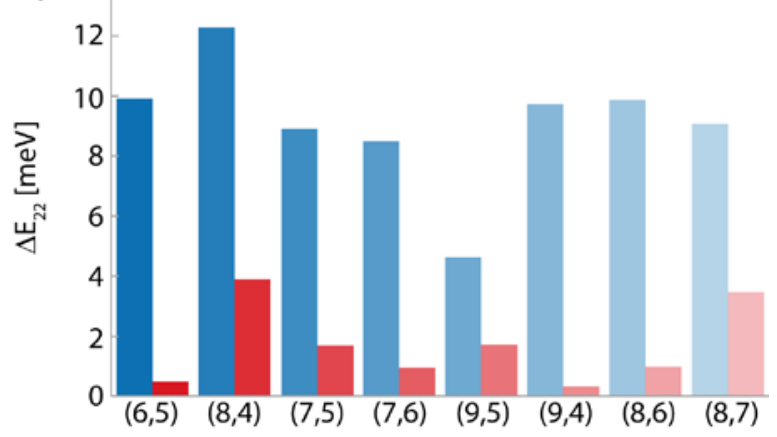

d)

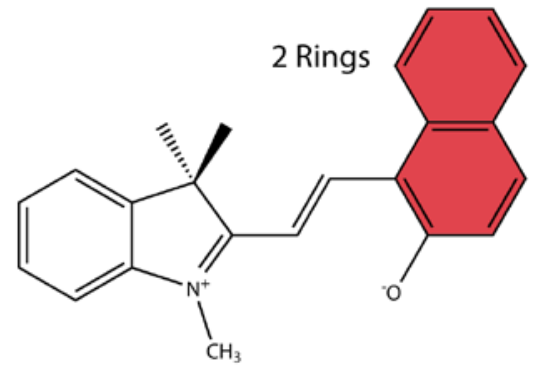

Figure 4 Shifts in transition energy for the one (blue) and two (red) ring spiropyran derivative for for a) $E_{11}$ transition energies and b) $E_{22}$ transition energies. Schematic illustration of the SP derivatives c) Spiropyran one aromatic ring, d) Spirooxazin -two aromatic Rings

molecule of comparable size, sketched in Fig. 4: i) spiropyran, which has only one phenyl ring in the benzopyran part, highlighted in blue in Fig. 4c), ii) spirooxazine, which has two phenyl rings, highlighted in red in Fig. 4d). All derivatives undergo the ring opening and closing isomerization. By increasing the amount of rings, the structures get more and more extended and the dipole moment becomes smaller (13.9 D for spiropyran, 6.64 $\mathrm{D}$ for spirooxazine) in the ring open form [31]. Those derivatives thus allow us to change the strength of the dipole moment while keeping orientation and coverage constant. The bar chart in Fig. $4 \mathrm{a}$ ) shows the shifts of the $\mathrm{E}_{11}$ transition energies and Fig $4 \mathrm{~b}$ ) the shifts of the $E_{22}$ transition energies of the different tubes $(150 \mu \mathrm{mol})$ for the different switches in their mero form. The biggest shift is observed for the one-ring derivative, whereas the switches with lower dipole moments induce smaller shifts. This supports the prediction that the change in exciton energy is proportional to the magnitude of the dipole.

\section{Improving the micelle filling and composition}

We further improve our samples and studied the influence of the temperature on the production of our SP/MC-CNTs hybrids. At higher temperatures the micelles expand [32], giving the switches more space to re-arrange, improving the homogeneity of the switch coating on the tubes' surface, hence optimizing the micelle swelling process. To prevent the solvent to evaporate before entering the micelles we worked below the boiling point of THF $\left(60^{\circ} \mathrm{C}\right)$, namely at $30^{\circ} \mathrm{C}, 40^{\circ} \mathrm{C}$, and $55^{\circ} \mathrm{C}$. Fig. 5a) displays the influence of the temperature on the emission intensity of the resulting UV samples. The plain micelle-encapsulated tubes without any switch show a decrease in intensity with increasing temperature. All chiralities behave in the same manner, as it can be seen in the first column of Fig. 5a). The other two columns depict the behavior for sample with different amount of switches, $100 \mu \mathrm{mol} / \mathrm{L}$ in Fig 5a) middle 
a)

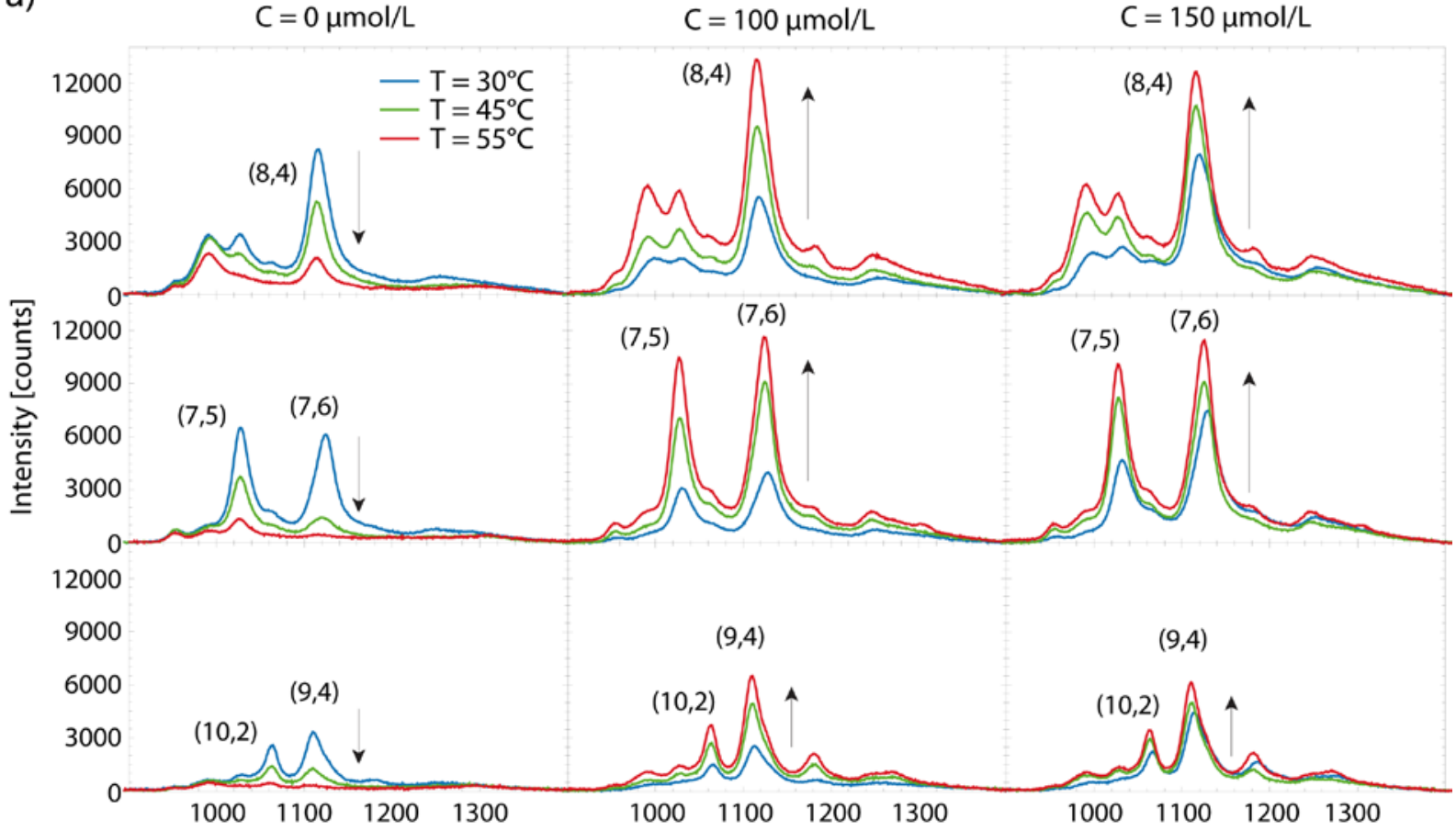

b)

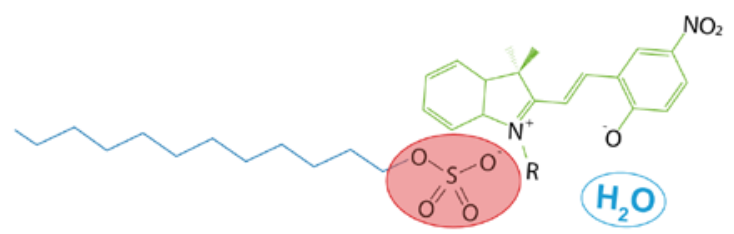

c)

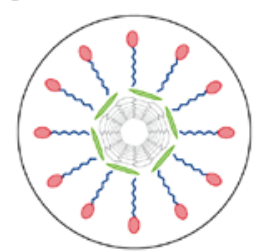

Room temperature

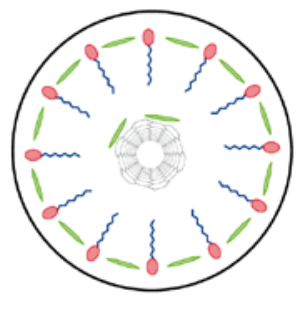

$55^{\circ} \mathrm{C}$

Figure 5 a) Photoluminescence intensities of the samples prepared under UV radiation. The different producing temperatures $\left(\mathrm{T}=30^{\circ} \mathrm{C}, 45^{\circ} \mathrm{C}, 55^{\circ} \mathrm{C}\right)$ are indicated through the different plotting colours. From left to right the intensities of the different concentrations are displayed (c $=0 \mu \mathrm{mol} / \mathrm{L}, 100 \mu \mathrm{mol} / \mathrm{L}, 150 \mu \mathrm{mol} / \mathrm{L}$ ). From top to bottom the different chiralities corresponding to the different excitation energies $\left(\lambda_{22}=590 \mathrm{~nm}\right.$, $650 \mathrm{~nm}, 730 \mathrm{~nm}$ ) are shown. Additional mechanism influencing the micelle swelling process: b) Schematic structure of the merocyanine- SDS ion pair with a water molecule as stabilization. c) Schematic structure of the new SDS-switch hybrid micelle. Due to the incorporation of the switch into the micelle less switch content is in close proximity of the CNTs.

panel and $150 \mu \mathrm{mol} / \mathrm{L}$ switch concentration in Fig 5a) right panel. Those samples show a distinct increase in intensity for higher production temperatures. This behavior can be explained when considering the micellization process. An increase in temperature results in a more pronounced movement of the SDS molecules making it more difficult for the anionic molecule to remain encapsulated within the micellar core. Some micelle break, triggering the CNTs re-bundling or precipitation, resulting in quenched luminescence. The increase in intensity of the UV samples, on the other hand, indicates a stabilization of the micelles. The presence of the dipole moment of the switch reduces the polarity of the surfactants by forming MC-SDS ion pairs (Fig. 5c), making the micelle less ionic [33]. The size of nonionic micelles enlarges with increasing temperature, thus more and more switches content is able to stabilize and penetrate the micelle [32]. Fig. 5c) sketch the stabilization 
a) Dark

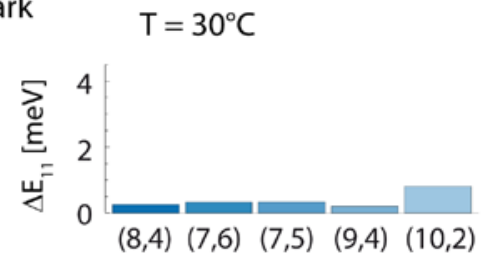

UV

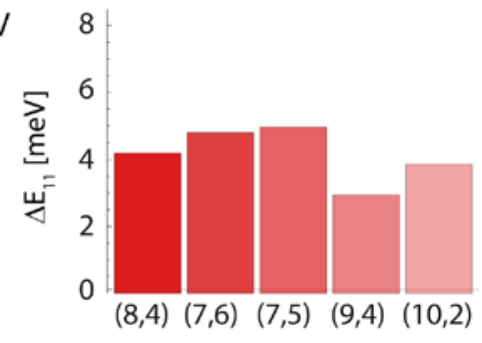

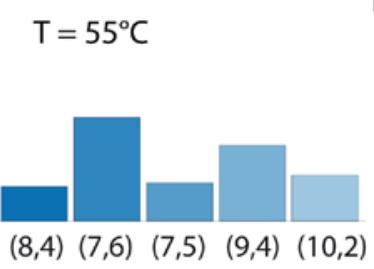

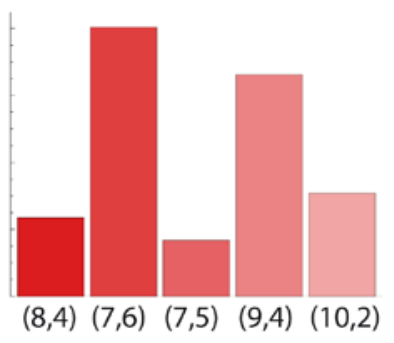

b) $\quad 0.25$ Dark

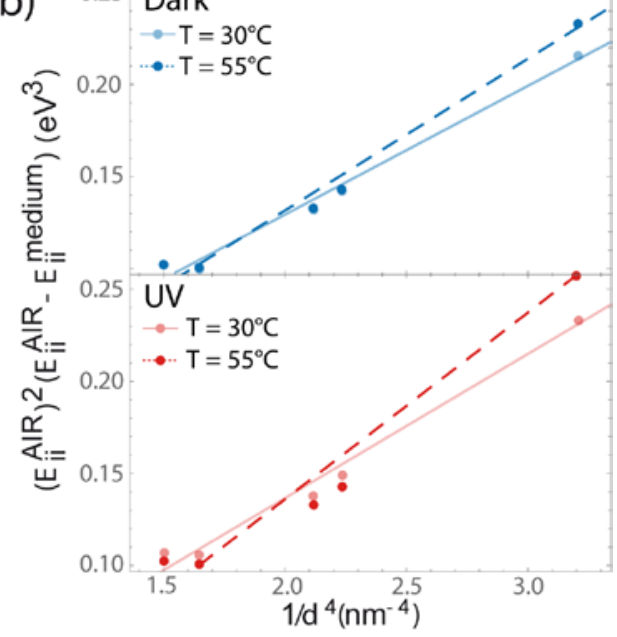

Figure 6 a) Comparison of the shifts for different tube species between DARK and UV samples with a concentration of $150 \mu \mathrm{mol} / \mathrm{L}$ prepared at $30^{\circ} \mathrm{C}$ (left panel) and $55^{\circ} \mathrm{C}$ (right panel). b) Solvatochromic shifts of the $\mathrm{E}_{11}$ transition of the $150 \mu \mathrm{mol}$ samples prepared at $30^{\circ} \mathrm{C}$ and $55^{\circ} \mathrm{C}$.

process of the expanded outer shell: The SDS-MC ion pair, the water molecule between the methylgroup on the indolinic part, and the oxygen of the benzopyran moiety stabilizes the ion pairs.

Even if higher temperatures expand the micelles, competitive processes lower the composition of the final structures and only a tiny fraction of the switches is now penetrating and remaining within the micelle core. The other fraction will stabilize the hydrophilic shell of the micelle, resulting in a reduction of the overall dipole coverage of the tubes and thus affecting the shifts magnitude.

The change of composition and morphology of the micelles swelled at higher temperatures changes the phenomenology of the optical response of the samples. The PLE measurements of the higher temperature samples display a different behavior than samples prepared at room temperature. With an increase in temperature, we observe that the shifts do not correlate any more to the switch concentration, they rather depend upon the tube diameter, the larger the diameter the greater the shift. This behavior is depicted in Fig. 6a) for the $150 \mu \mathrm{L}$ concentrated DARK and UV samples prepared at $55^{\circ} \mathrm{C}$ and compared to the $30^{\circ} \mathrm{C}$ ones. A similar but much weaker behavior is also observed for the other samples at higher temperatures and even at lower switch concentrations. It is worth noting that such diameter-dependent shift was not observed for the samples prepared at room temperature. The difference in the phenomenology is ascribed to the fact that by increasing the temperature, the separation between the tubes and the switches increases. This reduces their mutual Coulomb interaction. At higher temperatures, the MC dipoles are localized in the outer shell of the micelles, so far away to make the dipole-dipole induced shifts negligible and no constant dipole-induced shifts can be observed.

The shifts we experimentally observe nicely follow the trend described by the Choi and Strano model for solvatochromic effects on CNTs [34]. Those shifts in the emission and absorption of a nanosystem originate from changes in the dielectric environment [35]. 
Dipole-Switch Induced Modification of the Emissive Response of Carbon Nanotubes

\begin{tabular}{|c|c|c|}
\hline $\mathbf{3 0}^{\circ} \mathbf{C}$ & $\mathrm{E}_{11}\left(\mathrm{eV}^{3} \mathrm{~nm}^{-4}\right)$ & $\mathrm{E}_{22}\left(\mathrm{eV}^{3} \mathrm{~nm}^{-4}\right)$ \\
\hline DARK & 0.074 & 0.27 \\
\hline UV & 0.076 & 0.29 \\
\hline
\end{tabular}

\begin{tabular}{|c|c|c|}
\hline $\mathbf{5 5}^{\circ} \mathbf{C}$ & $\mathrm{E}_{11}\left(\mathrm{eV}^{3} \mathrm{~nm}^{-4}\right)$ & $\mathrm{E}_{22}\left(\mathrm{eV}^{3} \mathrm{~nm}^{-4}\right)$ \\
\hline DARK & 0.082 & 0.28 \\
\hline UV & 0.101 & 0.30 \\
\hline
\end{tabular}

Table 1 Comparison of slopes obtained for the DARK and UV samples prepared respectively at $30^{\circ} \mathrm{C}$ and at $55^{\circ} \mathrm{C}$

Table 1 depicts the values for the DARK and UV samples prepared, respectively, at $30^{\circ} \mathrm{C}$ and at $55^{\circ} \mathrm{C}$. The values for the slope increase for the higher production temperature. The main changes can be observed in the emission $\left(E_{11}\right)$, e.g. the value for the $\mathrm{UV}$ sample rises from $0.076 \mathrm{eV}^{3} \mathrm{~nm}^{-4}\left(30^{\circ} \mathrm{C}\right)$ to $0.101 \mathrm{eV}^{3} \mathrm{~nm}^{-4}\left(55^{\circ} \mathrm{C}\right)$, concluding that the formation of the SDS-MC hybrid surfactant is changing the dielectric environment of the tubes leading to higher solvatochromic shift which are known to be diameter tube dependent [34].

The increase in temperature stabilizes the micelles by incorporating the switches into their outer hydrophilic shell, which lowers the number of molecules at the surface of the CNT. The dipole-dipole interaction of the tubes and the switches becomes negligible. We observe the solvatochromic shifts of the CNT transition energies due to the change of the dielectric environment. Working at room temperature, therefore, is the best configuration to ensure the switches are closest the to the CNT sidewall. It is thus the most beneficial for observing the dipole-dipole induced shifts in the transition energies for CNTs decorated with molecular switches.

\section{Conclusion}

We demonstrated that micelle swelling is a simple and successful method to investigate the interaction of the dipole switch spiropyran with carbon nanotubes. We verified the theoretical predictions on the effect of the dipole moment of merocyanine on the excitation and emission energies of the CNTs. To further validate the predictions, we compared the shifts for molecules with similar morphologies but different intensity of their dipole moments. We investigated the effect of the temperature on the hybrid production process to identify the preparation conditions with the highest dipole coating of the tubes.

\section{Acknowledgements}

This work has been funded by the DFG under the SfB 658, subproject A6. 
Dipole-Switch Induced Modification of the Emissive Response of Carbon Nanotubes

\section{References}

[1] Guo X, Huang L, O'Brien S, Kim P and Nuckolls C 2005 Directing and sensing changes in molecular conformation on individual carbon nanotube field effect transistors Journal of the American Chemical Society 127, 15045-15047.

[2] Glaeske M and Setaro A 2013 Nanoplasmonic colloidal suspensions for the enhancement of the luminescent emission from single-walled carbon nanotubes Nano Research 6, 593-601.

[3] Roquelet C, Garrot D, Lauret JS, Voisin C, Alain-Rizzo V, Roussignol Ph, Delaire JA and Deleporte E 2010 Quantum efficiency of energy transfer in noncovalent carbon nanotube/porphyrin compounds Appl. Phys. Lett. 97, 141918.

[4] Ernst F, Heek T, Setaro A, Haag R and Reich S 2013 Excitation characteristics of different energy transfer in nanotube-perylene complexes Appl. Phys. Lett. 102, 233105.

[5] Natali M and Giordani S 2012 Molecular switches as photocontrollable "smart" receptors Chem. Soc. Rev. 41, 4010.

[6] Fischer E and Hirshberg Y 1952 Formation of coloured forms of spirans by low-temperature irradiation Journal of the Chemical Society 4522-4524.

[7] Raymo FM, Alvarado RJ, Giordani S and Cejas MA 2003 Memory Effects Based on Intermolecular Photoinduced Proton Transfer JACS 125, 2361.

[8] R. Klajn 2014 Spiropyran-based dynamic materials Chem. Soc. Rev. 43, 148-184.

[9] Setaro A, Bluemmel P, Maity C, Hecht S and Reich S 2012. Non-Covalent Functionalization of Individual Nanotubes with Spiropyran-Based Molecular Switches Advanced Functional Materials 22, 2425-2431.

[10] Nish A, Hwang JY, Doig J and Nicholas RJ 2007. Highly selective dispersion of single-walled carbon nanotubes using aromatic polymers Nature nanotechnology 2, 640-646.

[11] Setaro A, Adeli M, Glaeske M, Przyrembel D, Bisswanger T, Gordeev G, Maschietto F, Faghani A, Paulus B, Weinelt M, Arenal R., Haag R and Reich S 2017 Preserving $\pi$-conjugation in covalently functionalized carbon nanotubes for optoelectronic applications Nature Communications 8, 14281.

[12] Bluemmel P, Setaro A, Popeney CS, Haag R and Reich S 2010 Interaction between singlewalled carbon nanotubes and alkyl polyglycerol derivatives Phys. Stat. Sol. B 247, 2891.

[13] Wang RK, Chen WC, Campos DK and Ziegler KJ 2008 Swelling the micelle core surrounding single-walled carbon nanotubes with water-immiscible organic solvents Journal of the American Chemical Society 130, 16330-16337.

[14] Bluemmel P, Setaro A, Popeney CS, Trappmann B, Haag R, and Reich S 2011 Amphiphile replacement on carbon nanotube surfaces: Effect of aromatic groups on the interaction strength Phys. Stat. Sol. B 248, 2532. 
Dipole-Switch Induced Modification of the Emissive Response of Carbon Nanotubes

[15] Matsuzawa M, Kato H, Ohyama H, Nishide D, Kataura H and Yoshida M 2011 Photoinduced Dispersibility Tuning of Carbon Nanotubes by a Water-Soluble Stilbene as a Dispersant Advanced Materials 23, 3922-3925.

[16] Kördel C, Setaro A, Bluemmel P, Popeney CS, Reich S and Haag R 2011 Controlled reversible debundling of single-walled carbon nanotubes by photo-switchable dendritic surfactants Nanoscale 4, 3029.

[17] Malic E, Setaro A, Bluemmel P, Sanz-Navarro CF, Ordejón P, Reich S and Knorr A 2012 Carbon nanotubes as substrates for molecular spiropyran-based switches Journal of Physics: Condensed Matter 24, 394006.

[18] Malic E, Weber C, Richter M, Atalla V, Klamroth T, Saalfrank P, ... \& Knorr A 2011. Microscopic model of the optical absorption of carbon nanotubes functionalized with molecular spiropyran photoswitches Physical review letters, 106, 097401.

[19] Setaro A, Kreft SK., Petersen MÅ, Nielsen MB and Reich S 2014 Optical properties of carbon nanotubes coated with orthogonal dipole switches Phys. Stat. Sol. B 251, 2356-2359.

[20] Bluemmel P, Setaro A, Maity C, Hecht S and Reich S 2012. Designing a spiropyran - based molecular switch for carbon nanotube functionalization: Influence of anchor groups and tubeswitch separation Phys. Stat. Sol. B 249, 2479-2482.

[21] Bluemmel P, Setaro A, Maity C, Hecht S and Reich S 2012 Tuning the interaction between carbon nanotubes and dipole switches: the influence of the change of the nanotube-spiropyran distance Journal of Physics: Condensed Matter 24, 394005.

[22] Roquelet C, Lauret JS, Alain - Rizzo V, Voisin C, Fleurier R, Delarue M, Garrot D, Loiseau A, Roussignol P, Delaire JA and Deleporte E 2010 П - Stacking Functionalization of Carbon Nanotubes through Micelle Swelling ChemPhysChem 11, 1667-1672.

[23] Kreft SK., Petersen MÅ, Nielsen MB, Reich S and Setaro A 2015 Isomerization of orthogonal molecular switches encapsulated within micelles solubilizing carbon nanotubes The Journal of Physical Chemistry C 119, 15731-15734.

[24] Glaeske M, and Setaro A 2014 Effect of hybrid isolation on the luminescence enhancement of carbon nanotube-gold nanorod composites Phys. Stat. Sol. B 251, 2480.

[25] Clave G, Delport G, Roquelet C, Lauret J-S, Deleporte E, Vialla F, Langlois B, Parret R, Voisin C, Roussignol P, Jousselme B, Gloter A, Stephan O, Filoramo A, Derycke V, and Campidelli S 2013 Functionalization of carbon nanotubes through polymerization in micelles: a bridge between the covalent and noncovalent methods Chemistry of Materials 25, 2700.

[26] Popeney CS, Setaro A, Mutihac RC, Bluemmel P, Trappmann B, Vonneman J, Reich S and Haag R 2012 Polyglycerol - Derived Amphiphiles for the Solubilization of Single - Walled Carbon Nanotubes in Water: A Structure-Property Study ChemPhysChem 13, 203-211. 
Dipole-Switch Induced Modification of the Emissive Response of Carbon Nanotubes

[27] Setaro A, Popeney CS, Trappmann B, Datsyuk V, Haag R and Reich S 2010 Polyglycerolderived amphiphiles for single walled carbon nanotube suspension Chemical Physics Letters 493, 147-150.

[28] Piantek M, Schulze G, Koch M, Franke KJ, Leyssner F, Krüger A, Navío C, Miguel J, Bernien M, Wolf M, Kuch W, Tegeder P and Pascual JI 2009 Reversing the thermal stability of a molecular switch on a gold surface: ring-opening reaction of nitrospiropyran Journal of the American Chemical Society 131, 12729-12735.

[29] Khairutdinov RF, Itkis ME and Haddon RC 2004 Light modulation of electronic transitions in semiconducting single wall carbon nanotubes Nano Letters 4, 1529-1533.

[30] Setaro A 2017 Advanced Carbon Nanotubes Functionalization. Journal of Physics: Condensed Matter 29, 423003.

[31] Zhang H, Guo X, Hui J, Hu S, Xu W and Zhu D 2011 Interface engineering of semiconductor/dielectric heterojunctions toward functional organic thin-film transistors Nano letters 11, 4939-4946.

[32] Liu R ed., 2008 Water-insoluble drug formulation CRC press.

[33] Metelitsa AV, Coudret C, Micheau JC and Voloshin NA 2014 Quantitative investigations of thermal and photoinduced J-and H-aggregation of hydrophobic spirooxazines in binary solvent through UV/vis spectroscopy RSC Advances, 4, 20974-20983.

[34] Choi JH and Strano MS 2007 Solvatochromism in single-walled carbon nanotubes Applied Physics Letters, 90, 223114.

[35] Setaro A, Popeney CS, Witt MU, Bluemmel P, Gläske M, Haag R and Reich S 2015 Chiral selectivity of polyglycerol-based amphiphiles incorporating different aromatic cores Phys. Stat. Sol. B, 252, 2536-2540. 